\title{
Arab Children's Reading Preference for Different Online Fonts
}

\author{
Asmaa Alsumait ${ }^{1}$, Asma Al-Osaimi ${ }^{2}$, and Hadlaa AlFedaghi ${ }^{2}$ \\ ${ }^{1}$ Computer Engineering Dep., Kuwait University, Kuwait \\ ${ }^{2}$ Regional Center For Development of Educational Software, Kuwait \\ alsumait@eng.kuniv.edu.kw, \{alosaimi,hadlaa\}@redsoft.org
}

\begin{abstract}
E-learning education plays an important role in the educational process in the Arab region. There is more demand to provide Arab students with electronic resources for knowledge now than before. The readability of such electronic resources needs to be taken into consideration. Following design guidelines in the e-learning programs' design process improves both the reading performance and satisfaction. However, English script design guidelines cannot be directly applied to Arabic script mainly because of difference in the letters occupation and writing direction. Thus, this paper aimed to build a set of design guidelines for Arabic e-learning programs designed for seven-to-nine years old children. An electronic story is designed to achieve this goal. It is used to gather children's reading preferences, for example, font type/size combination, screen line length, and tutoring sound characters. Results indicated that Arab students preferred the use of Simplified Arabic with 14-point font size to ease and speed the reading process. Further, 2/3 screen line length helped children in reading faster. Finally, most of children preferred to listen to a female adult tutoring sound.
\end{abstract}

Keywords: Child-Computer Interfaces, E-Learning, Font Type/Size, HumanComputer Interaction, Information Interfaces and Presentation, Line Length, Tutoring Sound.

\section{Introduction}

Ministries of education in the Arab region are moving toward adopting e-learning methods in the educational process. In fact, the public schools in the state of Kuwait are using e-learning programs for elementary and middle school students. These elearning programs are designed by the Ministry of Education and the Regional Center for Development of Educational Software (ReDSOFT). Nowadays e-learning became a necessity not a luxury as it affects the patterns of accumulating knowledge. Thus, without a sound educational model, e-learning education will fail the designer, the instructor and the learner. It is very important to design the e-learning material in a way that can keep children's concentration focused on the tasks. This can be done by using proper font type, size, and screen line length.

Many researchers have considered building a set of guidelines to be used in designing e-learning software for adults and children. For example, Bernard et. al. (2002) 
studied adults' preferences regarding font type and size from measuring adults' reading efficiency, adults' reading time, legibility, attractiveness, and adults' font preference [1]. They found that Times and Arial font type with size 12-point were read faster than other fonts and sizes that were tested. Further, Arial and Courier font type were considered the most legible fonts. Again, Arial font type with 12-point size was the most preferable to read online. Another study carried out for adults compared the fonts used in previous editions of Windows with those new created for Windows Vista [3]. They found that the new fonts designed for Windows Vista such as Cambria and Constantia were more legible than traditional Times New Roman. While studies conducted on children indicated that the children prefer to read English text that is Arial font type with 14-point size and Comic font type with 12-point size [2]. Moreover, in English, adults prefer to read medium line length (approximately 65 to 75 CPL) and children prefer narrow line length (approximately 45 CPL) [4, 6].

On the other hand, few studies were conducted to find adult users' preferences for Arabic script. For example, Hemayssi et. al. concluded that in order to increase legibility, it is preferred to use bold fonts, colors and clear icons [5].

Most existing research in this area is oriented to build guidelines for designing English e-learning programs. Therefore, in this paper, we examined the preferences of seven to nine years old Arab children's for five font types at sizes 12- and 14-point and their screen line length using an electronic story.

\section{Usability Evaluation Process}

The usability evaluation of the Arabic electronic story for font type, size, and line length was conducted on July 2008. Participants were asked to spend half an hour with the e-story under the supervision of the ReDSOFT team member experimenter. At the beginning of the usability evaluation, participants were asked to complete a user profile questionnaire. The background questionnaire revealed that thirty five participants (16 males, 19 females) aged between seven and nine years old. Moreover, majority $(86 \%)$ of the participants use computer at home, and almost all of them $(97 \%)$ had previous experience with electronic reading.

Each page of the e-story was written with a different combination of font type and size. In total, the e-story was presented in 10 pages representing the five font types (Arial Unicode MS, Courier New, Microsoft Sans Serif, Simplified Arabic, and Traditional Arabic) and two font sizes (12 and 14). Figure 1 shows sample of a page of the e-story.

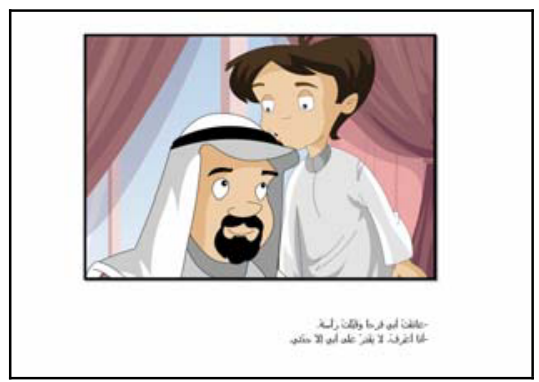

Fig. 1. A sample of a page of the e-story 
After reading each page of the e-story, participants were asked to answer four questions to measure their satisfaction regarding font type and size used. 1. Is this font type and size easy to read? 2. Do you think you can read faster with this font type and size? 3. Is this font type and size attractive? 4. Would you like this font type and size to be used in your schoolbook?

Text was displayed in the center of the screen. Fonts were black presented on a white background. A stopwatch was used to record the time participants took to read the paragraphs and the experimenter also noted the number of incorrectly pronounced words.

To confirm findings, participants were asked to read a simple sentence with all font type and size combinations in a single page and select their preferable font type and size.

Also participants were asked to choose the most comfortable screen line length while reading. The same font type and size (Simplified Arabic with size 14-pionts) script was presented to the participant in different line length conditions; $1 / 3$ screen line length, 2/3 screen line length, and full screen line length. The participant was asked to rate his/her reading comfortance and satisfaction with the three different screen line lengths.

We also explored a new direction for e-learning computer tutoring sound characters, which we believe will maximize students' learning gains and enjoyment. The traditional scenario allows students to interact primarily with a single coach or tutor character sound on-screen. In this evaluation process, we allowed the child to select his own tutor sound to be a teacher, a boy, or a girl.

The usability evaluation process measured seven main factors in the reading process: the ease of reading factor, reading faster factor, font attractiveness factor, the desire to use font combination in schoolbooks factor, the font preferences factor, the screen line length factor. And finally the tutor character sound factor. Table 1 shows different font types and sizes used in the experiment.

Table 1. Font types and sizes studied

\begin{tabular}{|c|c|c|}
\hline Font type & 12-point size & 14-point size \\
\hline Arial Unicode MS & أنا أحب القراءة & أنا أحب القراعة \\
\hline Courier New & 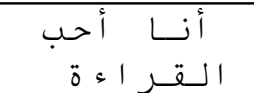 & أنــا أحسب الـقــــ اءة \\
\hline Microsoft Sans Serif & أنا أحب القراءة & أنا أحب القراءة \\
\hline Simplified Arabic & أنا أحب القراءة & أنا أحب القر اءة \\
\hline Traditional Arabic & أنا أحب القراءة & أنا أحب القر اعة \\
\hline
\end{tabular}

\section{Results}

\subsection{Ease of Reading}

Ease of reading measures whether a specific font type and size gave children an impression that the reading process was simple and trouble free. Analysis showed that 
$61 \%$ of the participants considered the Simplified Arabic font as an easiest to read font. The lowest ease of reading score was given to the Courier New and Traditional Arabic.

Further analysis is done to find font sizes that help children in reading faster. The results showed that there is small difference between fonts that are sized 12-point and fonts that are sized 14-point. $57 \%$ of the children thought that 14-point sized font are easy to read in oppose to $45 \%$ who thought that 12 -point sized font is easy to read.

The results revealed that using Simplified Arabic with 14-point font size was considered the easiest to read font for Arab children.

\begin{tabular}{|l|}
\hline Guideline 1 \\
\hline $\begin{array}{l}\text { Use Simplified Arabic with 14-point font size to ease the } \\
\text { reading for Arab children. }\end{array}$ \\
\hline
\end{tabular}

\subsection{Reading Faster}

The second factor to be measured was reading speed. It is a variable that measures whether a specific font type and size gave children an impression that the reading process was done quicker. Analysis showed that font type has an affect on children's reading speed. Simplified Arabic and Microsoft Sans Serif had the highest participants' score preference; $54 \%$ for reading faster. On the other hand, Courier New had the lowest preference score regarding the reading faster factor, only $34 \%$ of the participants thought that they can read faster with it.

When it comes to font size, results showed that children (47\%) had preferred to read fonts that are sized 14-point. Fonts with size 12-point got a lower preference score.

In addition, results showed that $60 \%$ of the participants believed that the Simplified Arabic with 14-point font size helped them read faster. In contrast, only $26 \%$ of the participants thought that Traditional Arabic with font size 12-point, will help them read faster.

\begin{tabular}{|l|}
\hline Guideline 2 \\
\hline $\begin{array}{l}\text { Use Simplified Arabic with 14-point font size to speed the } \\
\text { reading for Arab children. }\end{array}$ \\
\hline
\end{tabular}

\subsection{Font Attractiveness}

Font Attractiveness measures whether children found a specific font type and size nice looking and eye catching. $66 \%$ of the participants considered the Arial Unicode MS as the most attractive font type among the types we tested.

When it comes to font size, results showed that it has no difference in attractiveness scores. Font sizes 12-point and 14-point had similar attractiveness scores (around $60 \%)$.

According to experimental results, Arial Unicode MS with 14-point font size got the best score when it comes to font type/size attractiveness. 
Guideline 3

Use Arial Unicode MS with 14-point font size when considering font attractiveness for children.

\subsection{Desire to Use Font Type and Size Combination in Schoolbooks}

Desire to use font combination in schoolbooks factor measures whether children want to use a specific font type and size in their schoolbooks. The results showed that Simplified Arabic with font size 12-point and Arial Unicode MS with font size 14-point are considered the most desired font type and size combinations preferred to be used in schoolbooks.

\section{Guideline 4}

Use Simplified Arabic with font size 12-point or Arial Unicode MS with font size 14-point when considering children's preferences for schoolbooks.

\subsection{Confirm Font Preference}

\subsubsection{2-Point Font Size}

Figure 2 shows font type preferences percentage with size 12-point. Arial Unicode MS was selected as the most preferred (45\%) font type with size 12-point.

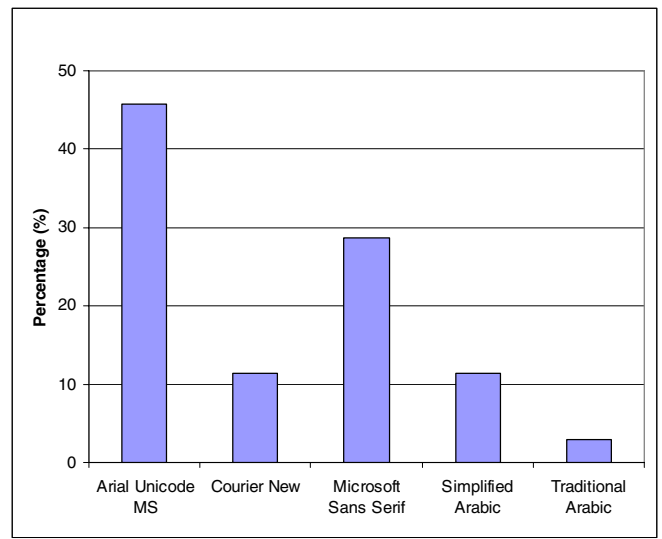

Fig. 2. The font type preference percentage with size 12-point

\subsubsection{4-Point Font Size}

Figure 3 shows font type preferences percentage with size 14-point. Arial Unicode MS was considered to be the most preferred (57\%) font type with size 14-point. 


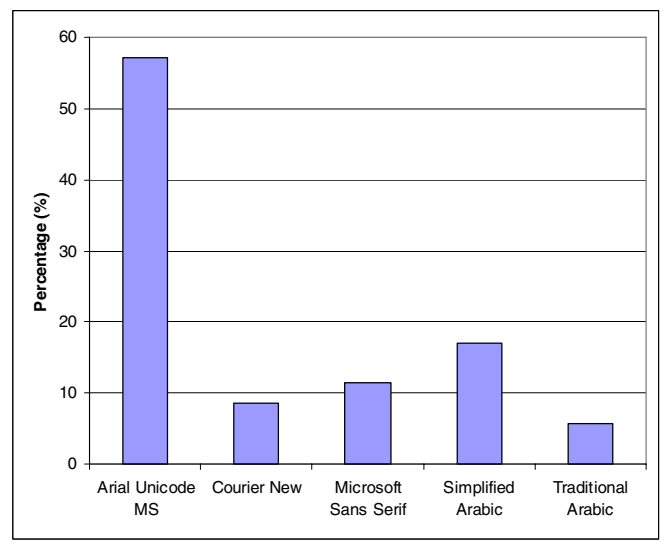

Fig. 3. The font type preference percentage with size 14-point

Figure 4 shows which font size is preferred by participants. Result showed that participants preferred to read using 14-point font size. These results are in agreement with the results obtained through the usability evaluation process of reading the estory.

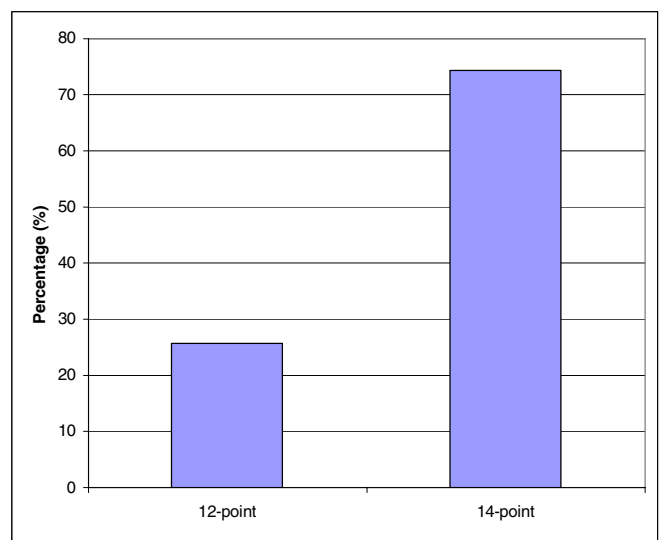

Fig. 4. Best font size selected by participants

\subsection{Tutoring Sound}

At the end of the experiment, we asked the child to choose a character sound to tutor $\mathrm{him} /$ her through the final part of the survey. Figure $5(\mathrm{a}, \mathrm{b}, \mathrm{c})$ shows the percentage of the selected tutoring sound character by the participants. The result showed that most children (48\%) regardless of their gender had selected the female teacher as a sound tutoring (Figure 5.a). This result is related to the fact that at this specific age (seven to nine years old) children are used to be taught by female teachers at the schools. 


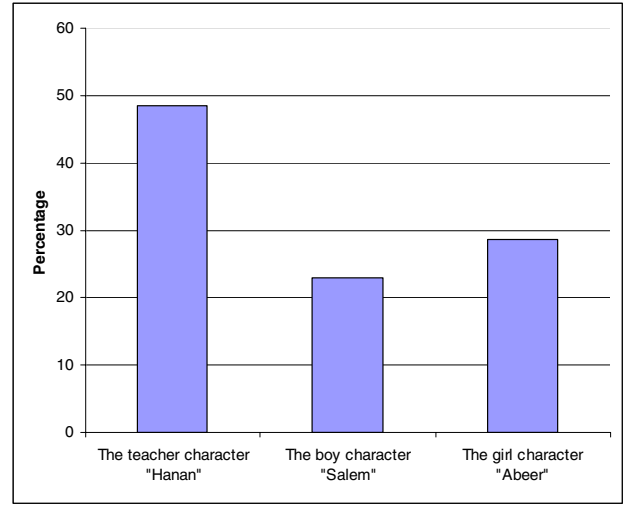

Fig. 5a. The percentage of the selected tutoring sound character by all participants

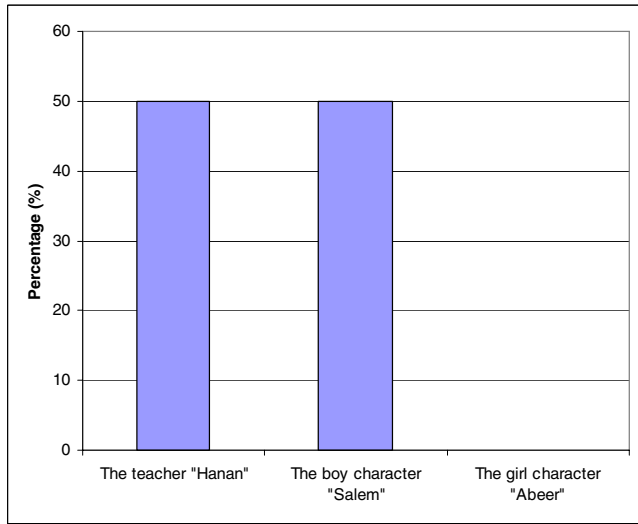

Fig. 5b. The percentage of the selected tutoring sound character male participants

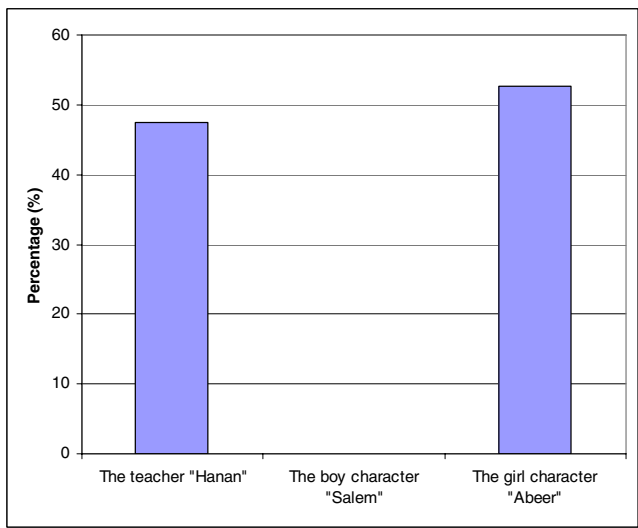

Fig. 5c. The percentage of the selected tutoring sound character by female participants 
Interestingly, results also reveled that the male child participants have selected only the boy sound charter "Salem" (50\%) along with the teacher sound character "Hanan" $(50 \%)$ (Figure 5.b). Similarly, the female child participants have selected only the girl sound character "Abeer" (53\%) along with the teacher character "Hanan" (47\%) (Figure 5.c). This indicates that children prefer only the teacher sound character along with the child tutor sound of the same gender.

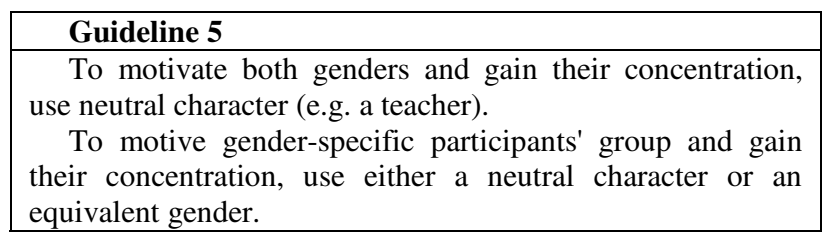

\subsection{Line Length}

This research also investigated the effect of screen line length on reading performance and satisfaction. The same paragraph was presented in three different screen line lengths. Despite the fact that there were no significant differences in satisfaction scores (see Figure 6), a line length that supports faster reading could impact the overall experience of e-learning programs. Reading rates were found to be the fastest $(4.59 \mathrm{sec} /$ word $)$ with the $2 / 3 \mathrm{screen}$ line length, $(5.44 \mathrm{sec} /$ word $)$ with the full screen line length and to be the slowest $(6.56 \mathrm{sec} /$ word $)$ at the $1 / 3$ screen line length.

Participants reported either liking or disliking the extreme screen line lengths $(1 / 3$ and full line length). Those that liked the 1/3 screen line length indicated that the short line length helped faster reading and was easier because it required less eye movement and the paragraph seemed neat and clear. Those that liked the full screen line length stated that they liked having more information on one line and believe that it seemed to them that they are reading less information.

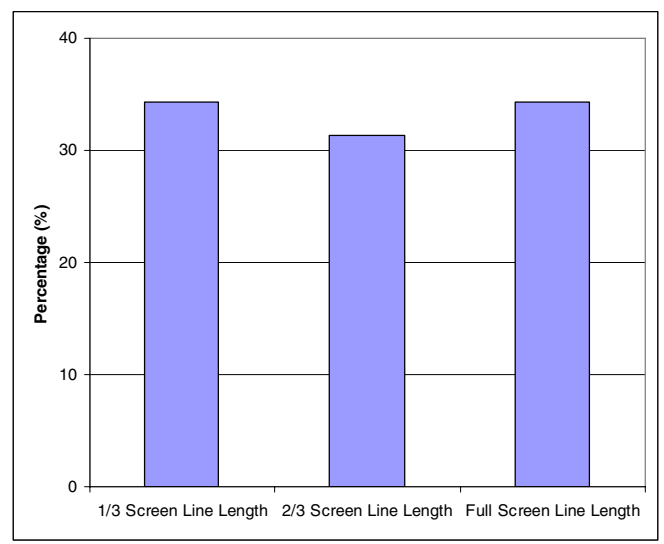

Fig. 6. The percentage of the preferred screen line length 
Although some participants reported that they felt like they were reading faster at $1 / 3$ screen line length, this condition actually resulted in the slowest reading speed. From this study, it may be beneficial to use $2 / 3$ line lengths when possible.

\section{Guideline 6}

Use $2 / 3$ screen line length to speed the reading.

\section{Conclusion}

Readable and satisfying e-learning interfaces are achieved through following e-learning design guidelines. These design guidelines need to be configured to suite some language characteristics. Therefore, this paper aimed to build a set of design guidelines for Arabic e-learning programs designed for seven-to-nine years old children.

The results of evaluating the e-story indicated that children preferred to read text using Simplified Arabic or Arial Unicode MS with size 14-point. These font type and size combinations were considered, among the different font type and size combinations used in this study, to be the easiest and fastest font type and size combinations to read. Conversely, children, for font attractiveness, chose other preference. They preferred to read using Arial Unicode MS with size 14-point. Generally, font types with 14-point size were preferable over the other font types with 12-point size. In addition, participants were able to read faster with $2 / 3$ screen line length.

When it comes to the tutoring sound character, children preferred to listen to a female adult tutoring sound, which is closer to their actual life style. Tutoring sound characters can play a critical success factor in learner acceptance of e-learning programs.

Larger combinations of font types and sized need to be assessed by Arab children. Further assessments are also needed to investigate the influence of other variables on the reading experience. Examples of those variables are: text color, effect of emphasizing pieces of text; bold, italic or underlined text, paragraph spacing, eye movements, and scrolling movements.

\section{References}

1. Bernard, M.L., Lida, B., Riley, S., Hackler, T., Janzen, K.: A comparison of popular online fonts: Which size and type is best?. Usability News. 4.4 (2002)

2. Bernard, M.L., Melissa, M., Talissa, F., Jan, M.: Which Fonts do Children Prefer to Read Online?. Usability News. 3.1 (2001)

3. Chaparro, B., Dawn Shaikh, A., Chaparro, A.: Examining the Legibility of Two New ClearType Fonts. Usability News. 8.1 (2006)

4. Dyson, M.C.: How physical text layout affects reading from screen. Behaviour \& Information Technology 23(6), 377-393 (2004)

5. Hemayssi, H., Sanchez, E., Moll, R., Field, C.: Designing an Arabic user experience: methods and techniques to bridge cultures. In: Proceedings of the Conference on Designing for User Experiences DUX 2005 (2005)

6. McPherson, M.A., Nunes, J.M., Zafeiriou, G.: New Tutoring Skills for Online Learning: Are e-Tutors adequately prepared for e-learning delivery? In: Proceedings of EDEN 2003 The Quality Dialogue; Integrating Quality Cultures in Flexible, Distance and e-learning, Rhodes, Greece, June 15-18, 2003, pp. 347-350 (2003) 